\title{
Repetitive transcranial magnetic stimulation for generalised anxiety disorder: a pilot randomised, double-blind, sham-controlled trial*
}

Gretchen J. Diefenbach, Laura B. Bragdon, Luis Zertuche, Christopher J. Hyatt, Lauren S. Hallion, David F. Tolin, John W. Goethe and Michal Assaf

\section{Background}

Repetitive transcranial magnetic stimulation (rTMS) holds promise for treating generalised anxiety disorder (GAD) but has only been studied in uncontrolled research.

\section{Aims}

This is the first randomised controlled trial (clinicaltrials.gov: NCT01659736) to investigate the efficacy and neural correlates of rTMS in GAD.

\section{Method}

Twenty five participants (active $n=13$; sham, $n=12$ ) enrolled rTMS was targeted at the right dorsolateral prefrontal cortex (DLPFC, $1 \mathrm{~Hz}, 90 \%$ resting motor threshold).

\section{Results}

Response and remission rates were higher in the active $v$. sham groups and there were significant group $\times$ time interactions for anxiety, worry and depressive symptoms, favouring active $v$. sham. In addition, right DLPFC activation during a decision-making gambling task increased at post-treatment for active rTMS only, and changes in neuroactivation correlated significantly with changes in worry symptoms.

\section{Conclusions}

Findings provide preliminary evidence that rTMS may improve GAD symptoms in association with modifying neural activity in the stimulation site.

\section{Declaration of interest}

G.J.D and J.W.G. receive material support from Neuronetics. J.W.G. has received speaker fees to discuss TMS at professional conferences from Neuronetics and grant support from Astra Zeneca, Bristol-Myers Squibb, Eli Lilly, Eisai, Forest, Hoffmann-La Roche, Janssen, Johnson \& Johnson, Merck/Schering-Plough, Neuronetics, Neosync, Novartis, Otsuka, Pfizer, Shire Sunovion, Takeda and Teva. D.F.T. receives funding from Palo Alto Health Sciences.

\section{Copyright and usage}

(c) The Royal College of Psychiatrists 2016.
Generalised anxiety disorder (GAD) is characterised by excessive and uncontrollable worry and central nervous system hyperarousal. $^{1}$ Prevalence is $5.7 \%$ in epidemiologic surveys. ${ }^{2}$ GAD is typically chronic, is associated with increased risk for functional and medical disability, ${ }^{3}$ and is the most costly of all the anxiety disorders. ${ }^{3}$ Although there are empirically supported treatments (pharmacotherapy; cognitive-behavioural therapy), up to half of patients do not benefit ${ }^{4,5}$ - a finding that highlights the importance of pursuing novel treatments for GAD.

Repetitive transcranial magnetic stimulation (rTMS) is a neuromodulation therapy that has been studied increasingly in recent years as a treatment for a variety of psychiatric disorders. During rTMS a magnetic coil is placed near the scalp to alter the electrical activity of brain regions and associated circuits. High-frequency $(>5 \mathrm{~Hz})$ pulses excite and low-frequency $(<1 \mathrm{~Hz})$ ones inhibit the adjacent cortex, with more complex activation and network connectivity alterations occurring in more remote brain regions. ${ }^{6,7}$ The original US Food and Drug Administration (FDA) indication was for treatment-resistant major depressive disorder using high-frequency pulses over the left dorsolateral prefrontal cortex (DLPFC). Meta-analytic research supports the efficacy of these stimulation parameters for depression (see for example Berlim et $a l^{8}$ ) as well as alternative parameters at the DLPFC, such as low-frequency right-sided stimulations. ${ }^{9}$ Research suggests that anxiety symptoms also improve in patients with

*Data from this study were presented at the annual meeting of the American Psychiatric Association New York, May 2014 and the meeting of the Society for Biological Psychiatry, New York, May 2014. major depressive disorder following rTMS. ${ }^{10}$ However, little is known about the use of rTMS to treat anxiety disorders, and no randomised controlled trials (RCTs) have investigated the efficacy of rTMS for GAD.

GAD is characterised by abnormal fronto-limbic circuitry, ${ }^{11,12}$ supporting the potential use of rTMS to target this circuit. A variety of symptom provocation tasks have been used to study functional brain activity in GAD. For example, worry, the hallmark symptom of GAD, is associated with increased activation in the prefrontal cortex (PFC), and decreased activation in amygdala, in both patients with GAD and healthy controls; however, this worry-related neural activity continues only in patients with GAD even after the worry-induction period has ended. ${ }^{13}$

The precise biological mechanism by which rTMS improves psychiatric symptoms remains poorly understood. It is possible that there is a direct effect on neural networks thereby improving emotion regulation processes. For example, in healthy volunteers, DLPFC stimulation alters activation of, and functional connectivity between, the DLPFC and ventromedial PFC (VMPFC) during emotional decision-making. ${ }^{14}$ Additional proposed biological mechanisms include epiphenomena such as normalisation of neuroendocrine and/or neurotrophic factors, ${ }^{15}$ which have also been shown to change via DLPFC neuromodulation in healthy volunteers (see for example Baeken et $\mathrm{al}^{16}$ ). To date there has been only one published open trial of rTMS for treating GAD. ${ }^{17}$ Ten patients completed six sessions (twice weekly for 3 weeks) of low-frequency rTMS at the right DLPFC. Functional neuroimaging during a gambling decision-making task was used to 
select the DLPFC stimulation site, and right DLPFC activation was evident for all ten participants. At post-treatment, $60 \%$ of the patients met criteria for remission (Hamilton Rating Scale for Depression $<8$ and Clinical Global Impression-Improvement $\leqslant 2) .{ }^{17}$ Results were largely maintained at 6-month follow-up. ${ }^{18}$ Although these outcomes are encouraging, only limited conclusions can be drawn given the absence of a control group. The current study is the first RCT for rTMS treatment for GAD (clinicaltrials.gov: NCT01659736). Low-frequency stimulation was targeted at the right DLPFC, located using structural neuronavigation. The number and timing of treatment sessions was chosen to align with the FDA-approved protocol for depression to protect against null effect because of inadequate dose. It was predicted that for patients receiving active rTMS there would be evidence of higher rates of treatment response and remission and larger improvements in anxiety, worry and depressive symptoms compared with patients undergoing placebo rTMS (using a 'sham' coil). In addition this study sought to explore the impact of neuromodulation on DLPFC activation and its association with symptom change. A leading cognitive theory suggests that GAD is characterised by intolerance of uncertainty - wherein uncertain or ambiguous situations create intense emotional responses that precipitate worry. ${ }^{19}$ Based upon this theory we chose to study neuroactivation in GAD under conditions of stressful uncertainty using a gambling decision-making task. This task was adapted from the task employed in previous rTMS GAD research, where it was shown to reliably activate DLPFC. ${ }^{17}$ It was hypothesised that there would be a larger change in DLPFC activations in the active $v$. sham group and that this change in DLPFC activation would correlate significantly with symptom improvements.

\section{Method}

\section{Participants}

Participants were adults (age $\geqslant 18$ ) diagnosed with GAD as the principal or coprincipal disorder of at least moderate severity (Clinical Global Impression - severity scale (CGI-S) $\geqslant 4$ ). ${ }^{20}$ Additional symptom inclusion criteria were similar to those used in the previous open trial of rTMS for GAD: ${ }^{17}$ the Hamilton Rating Scale for Anxiety (HRSA) $)^{21} \geqslant 18$ and 17-item Hamilton Rating Scale for Depression $(\mathrm{HRSD})^{22} \leqslant 17$. Exclusion criteria were brain trauma or disorder; serious and/or unstable medical illness (for example cardiac disease, thyroid disease); posttraumatic stress disorder (current); substance use disorder (past 6 months); lifetime bipolar, psychotic, developmental or obsessive-compulsive disorder; judged too psychiatrically unstable to participate (for example acute suicidality); any contraindication for magnetic resonance imaging (MRI, such as metal in the body) and/or rTMS (such as history of epilepsy); and concurrent psychotherapy. Concurrent pharmacotherapy was stabilised (type and dose) for 3 months prior to study entry with the exception of benzodiazepines as needed, which were stabilised on a daily dose for at least 2 weeks (based on the medication half-life). Patients were required to keep medication use stable throughout the treatment phase of the study and weekly assessments via interview confirmed that all patients adhered to this requirement.

Participants were recruited through an out-patient clinic specialising in the treatment of anxiety and related disorders, as well as newspaper advertisements, internet (for example Google ads, Craigslist, clinicaltrials.gov), community flyers, physician referral and media coverage. The study CONSORT diagram is presented in online Fig. DS1. Of the 34 patients who met study entry criteria, 8 withdrew prior to randomisation. Participants who withdrew prior to randomisation did not differ from those randomised on any pre-treatment clinical or demographic variable (all $P s$ >0.05). Of the 26 patients randomised to treatment, 14 were allocated to active rTMS and 12 to sham. However, data were excluded when patients adhered to the treatment schedule for fewer than 25 consecutive treatment sessions (i.e. there was a gap $\geqslant 7$ days in treatments sessions between sessions 6 and 26) resulting in exclusion of data from one patient (active). Thus, the final sample used in data analyses included 13 in the active and 12 in the sham group.

\section{Measures}

Diagnostic status was determined using the Mini-International Neuropsychiatric Interview (MINI). ${ }^{23}$ Principal/coprincipal diagnoses were determined using the Clinician's Severity Rating (CSR) with a 0 to 8 scale rated from 'absent' to 'very severe..24 The CGI-S was used to assess global illness (1, normal, not at all ill to 7 , extremely ill). The CGI - improvement scale (CGI-I) ${ }^{20}$ was rated from 1 very much improved to 7 , very much worse.

\section{Primary outcome measure}

The HRSA was the primary outcome measure and the structured interview guide was used. ${ }^{21}$ Responder status was defined as $\geqslant 50 \%$ HRSA improvement, and remission total HRSA $<8$ and a CGI-I score of 1 (very much improved) or 2 (much improved). These response and remission criteria were used in a previous study of rTMS for GAD. ${ }^{17}$

\section{Secondary outcome measures}

Self-reported worry was assessed using the Penn State Worry Questionnaire (PSWQ) ${ }^{25}$ and depression using the Depression Anxiety Stress Scales-Depression Subscale (DASS-DEP). ${ }^{26}$ Clinician-rated depression was assessed using the structured interview guide version of the 17 -item HRSD. ${ }^{27}$

\section{Treatment}

\section{rTMS}

Treatment was administered using the FDA-Cleared Neurostar TMS Therapy System, but applied non-FDA-approved location and intensity parameters for a non-FDA approved indication. rTMS was delivered at a frequency of $1 \mathrm{~Hz}$ for $15 \mathrm{~min}(900$ pulses/session) with the intensity at $90 \%$ of the resting motor threshold, to the right DLPFC, for 30 sessions (5 days/week for 6 weeks; 27000 total pulses).

\section{Sham}

Participants receiving sham rTMS completed the same procedures as those in the active rTMS group, but treatments were administered using the Neuronetics XPLOR coil. This sham coil looks and sounds like the active coil to preserve the doublemasking, but the intensity of the magnetic stimulus is far below the level needed to produce clinical benefit.

\section{Structural MRI and neuronavigation}

We obtained anatomical MRI brain images using a Siemens 3T Allegra MRI machine. $T_{1}$-weighted brain structure images were collected using a 3D MPRAGE pulse sequence (repetition time $(\mathrm{TR})=2300 \mathrm{~ms}$, echo time $(\mathrm{TE})=2.74 \mathrm{~ms}$, inversion time $(\mathrm{TI})=900 \mathrm{~ms}$, flip angle $8^{\circ}$, field of view $(\mathrm{FOV})=176 \times 256 \mathrm{~mm}$, matrix $176 \times 256 \times 176$, voxel size $1 \times 1 \times 1 \mathrm{~mm}$, pixel bandwidth $190 \mathrm{~Hz}$; total scan time $7 \mathrm{~min} 37 \mathrm{~s}$ ). 
The right DLPFC target for all patients was identified based on Montreal Neurological Institute (MNI) coordinates for the right DLPFC target $(x=42, y=36, z=32)$ provided by Bystritsky and colleagues $^{17}$ as the mean pre-treatment peak voxel from their group functional-MRI (fMRI) data in patients with GAD. A high-resolution single-subject structural scan in MNI space (Colin-27 template, ch2.nii, voxel size $1 \times 1 \times 1 \mathrm{~mm}$ ), along with a $3 \mathrm{~mm}$ radius target sphere image cantered at the right DLPFC MNI coordinate were non-rigidly coregistered to the patient's $T_{1}$ image in native space using the SPM8 normalise function. The normalise function thereby transformed the right DLPFC target sphere from its location in MNI space, to its corresponding location in patient native space.

A frameless stereotactic neuronavigation system (visor2, ANT Neuro, Enschede, Netherlands; http://www.ant-neuro.com) was used to guide the coil to the patient's right DLPFC brain target. The neuronavigation system was comprised of visor2 software running on a laptop computer (EliteBook 8560w, Hewlett Packard, Palo Alto, California, USA) connected to an infrared positioning camera (NDI, Waterloo, Ontario, Canada).

\section{fMRI task}

The fMRI task was adapted from a gambling task designed to induce anxiety related to uncertainty during decision-making. ${ }^{17}$ In the current study patients were presented with two cards - red $v$. blue - and instructed to 'look for a pattern' and predict which card would be drawn next. Patients were given 50 points and told that they could win or lose points ( 2 points per trial) based upon correct or incorrect predictions. No monetary value was associated with point wins or losses. Unknown to the participants, trials were presented in two blocks: win blocks ( $75 \%$ of the time the patient's choice is correct) and lose blocks (75\% of the time the patient's choice is incorrect). There were eight events in each block and six blocks of each condition. Given the set win/lose parameters, all patients ended with a total loss of 16 points. fMRI analyses were conducted on the completers sample ( $n=9$ active, $n=10$ sham), and excluded three additional patients in sham $(n=2$ ended the MRI prematurely; $n=1$ had incidental findings on structural MRI with no clinical manifestation). Thus, fMRI data were analysed for $n=9$ in the active group and $n=7$ in the sham group.

Blood oxygenation level-dependent (BOLD) contrast was obtained with $T_{2}{ }^{*}$-weighted echo planar imaging (EPI) sequence $(\mathrm{TR}=1860 \mathrm{~ms}, \mathrm{TE}=27 \mathrm{msec}$, flip angle $70, \mathrm{FOV} 22 \mathrm{~cm}, 64 \times 64$ acquisition matrix) with 36 contiguous axial functional slices of $3 \mathrm{~mm}$ thickness with $1 \mathrm{~mm}$ gap, yielding $3.4 \times 3.4 \times 4 \mathrm{~mm}$ voxels.

\section{Procedure}

Study procedures were approved by the Hartford Hospital Institutional Review Board (DIEF003523HI) and all patients gave written informed consent prior to participation. The CONSORT checklist is provided in online Fig. DS2. A licensed clinical psychologist completed masked assessments. Clinician and selfreports were collected at pre-, post- and 3-month follow-up, with a subset of measures collected weekly (sessions 1, 6, 11, 16, 21 and 30). The study design initially included a 6-month follow-up assessment, however, a new study was initiated part-way through the current trial offering active treatment for sham non-responders after the 3-month follow-up (clinicaltrials.gov: NCT01815099). Given that 6-month follow-up data were collected for only a subset of participants, 3-month follow-up was used as the end-point in the current analyses. Patients completed MRI at pre- and post-treatment. Adverse events were assessed using a checklist at each visit during the first 2 weeks of treatment, and weekly thereafter. Adverse events spontaneously reported were also recorded. In this parallel-group design, patients were randomised (1:1 ratio) using a computerised random number generator in groups of 10 for the first 20 participants. Once 20 participants were enrolled, a randomisation schedule was created to replace for attrition. Sample size was set a priori as 10 participants per group based upon feasibility for pilot study data collection. Initially patients were not going to be replaced for attrition; however, given the high drop-out rate the protocol was revised to replace for patient attrition. A licensed clinical psychologist who had no direct patient contact developed and held the randomisation schedule. The schedule was shared only with the rTMS technician responsible for coil selection. Thus, the treating psychiatrist, evaluator and patients were not informed of treatment condition assignment.

\section{Data analytic plan}

\section{Clinical outcomes}

Baseline demographic and clinical characteristics were compared by treatment group using between-group $t$-tests. Frequency counts of response and remission status were compared using chi-square. These analyses were conducted for treatment completers $(n=9 / 13$ and $n=10 / 12$ for active and sham, respectively) as well as an intent-to-treat (ITT) sample ( $n=13$ active and $n=12$ sham) including participants who had attended at least one rTMS session. Repeated measures analysis of variance (ANOVA) was used to determine changes in primary and secondary outcome measures. For these analyses a series of two group (active $v$. sham) $\times$ three time (pre-, post-, follow-up) ANOVAS were conducted for the ITT sample after using multiple imputation procedures $^{28}$ to replace missing data. We conducted ANOVAs for the completers sample as well. Results from the completers analyses differed from the ITT analyses primarily on the number of analyses reaching statistical significance, presumably because of lower power. For parsimony, only the ITT analyses are reported here. The completers analyses are available in the online supplement (Supplement DS1 and Tables DS1 and DS2). The primary result of interest is the group $\times$ time interaction. Statistically significant interactions were followed by within-group paired $t$-tests (preto-post, pre-to-follow-up) and effect sizes (Cohen's $d$, interpreted as 0.30 small; 0.50 medium; and 0.80 large $).{ }^{29}$ Given that this is a pilot study with small samples, statistical trends $(P<0.10)$ are also reported for hypothesis generation purposes. The frequencies of adverse events were compared using chi-square. For all chi-square analyses, results with at least one cell with $n<5$ participants should be interpreted cautiously.

\section{Imaging data analysis}

The fMRI data were processed using SPM8, including motion correction using the INRIAlign toolbox, normalisation to MNI template and smoothing $\left(5 \mathrm{~mm}^{3}\right.$ full-width at half maximum (FWHM) Gaussian kernel). Data were then analysed using a general linear model (GLM) approach. For each individual, the win and lose blocks were modelled as separate regressors. However, based on the previous results, ${ }^{17}$ a contrast defining both regressors as main effect (compared with baseline) was defined. Individual contrast images were entered into a group (active $v$. sham $) \times$ time (pre- $v$. post-treatment) repeated measure ANOVA to assess a group $\times$ time interaction. Since this report is focused on the activation change in the stimulation site, group results were masked with a customised right DLPFC BrainMap volume-of-interest (thresholded at 25\%). ${ }^{30}$ 


\section{Results}

\section{Pre-treatment characteristics and attrition}

Pre-treatment demographic and clinical characteristics for the ITT sample are presented in Table 1. The two groups were matched well on demographic variables and most clinical variables; however, patients randomly assigned to the active group presented with more severe anxiety and worry. One-third of patients randomised to the active group and one-fifth of those assigned to the sham group discontinued the study prior to completing 30 sessions. This difference was not statistically significant $\left(\chi^{2}(1, n=25)=0.68, P=0.409\right)$. Those patients who dropped out did not differ from those who completed treatment on any pre-treatment demographic or clinical variables. In addition, within the completers sample, the active and sham groups did not differ on pre-treatment demographic or clinical variables (online Table DS1).

\section{Responder and remitter status}

\section{Participants who completed treatment}

At post-treatment significantly more patients met responder status in the active $(7 / 9,77.8 \%) v$. the sham group $(2 / 10,20.0 \%)$ $\left(\chi^{2}(1, n=19)=6.34, P=0.012\right)$. A similar pattern emerged for remitter status at post-treatment (active group $=3 / 9,33.3 \%$, sham group $1 / 10,10.0 \%)$; however, this difference was not statistically significant $\left(\chi^{2}(1, n=19)=1.55, P=0.213\right)$. At 3-month follow-up there were significantly more responders $\left(\chi^{2}(1, n=18)=11.46\right.$, $P=0.001)$ and remitters $\left(\chi^{2}(1, n=18)=9.00, P=0.003\right)$ in the active $(7 / 9,77.8 \%$ responders; $6 / 9,67.7 \%$ remitters $) v$. the sham group $(0 / 10,0 \%$ responders; $0 / 9,0 \%$ remitters).

\section{Intent-to-treat analysis}

In the ITT analysis (using the last available assessment as endpoint) response rates were significantly higher in the active $v$. sham group (active $8 / 13,61.5 \%$, sham group 2/12, $16.7 \%$; $\left.\chi^{2}(1, n=25)=5.24, \quad P=0.022\right) \quad$ at post-treatment. However, remitter rates did not differ significantly in the active $(4 / 13$,
$30.8 \%) \quad v$. sham group $(1 / 12, \quad 8.3 \%) \quad\left(\chi^{2}(1, n=25)=1.96\right.$, $P=0.161)$ at post-treatment. At 3-month follow-up there were significantly more responders in the active $(8 / 13,61.5 \%) v$. the sham group $(0 / 12,0 \%)\left(\chi^{2}(1, n=25)=10.86, P=0.001\right)$ as well as significantly more remitters in the active $(7 / 13,53.8 \%) v$. the sham group $(0 / 12,0 \%)\left(\chi^{2}(1, n=25)=8.97, P=0.003\right)$.

\section{Primary and secondary outcomes}

Results for the primary and secondary outcomes in the ITT sample are presented in Table 2. Planned contrasts showed that for the HRSA, patients in both the active and sham groups experienced large and statistically significant improvements at post-treatment, but these gains were maintained only in the active group at follow-up. For all secondary symptom variables, only the active group demonstrated statistically significant improvements at post-treatment and follow-up assessments. Effect sizes for secondary symptoms ranged from moderate to large in the active and negligible to moderate in the sham group.

\section{Adverse events}

The frequency of adverse events was similar in the active and sham groups, with pin prick or pain at the stimulation site being the most commonly reported events (Table 3 ). The only statistically significant difference between groups was the presence of facial twitch. One serious adverse event occurred when a patient in the active group was admitted to hospital for evaluation of chest pain; however, the event was determined to be unrelated to the study intervention.

\section{fMRI}

A repeated measures ANOVA demonstrated a significant group $\times$ time interaction in the right DLPFC $(x=42, y=41, z=25$; $F_{(1,56)}=8.07, P=0.006$; online Fig. DS3) such that activation in this region significantly increased after active treatment $(t(1,8)=-3.65, P=0.006)$ and tended to decrease after sham treatment $(t(1,6)=2.104, P=0.08)$. Moreover, the changes in right DLPFC activation correlated significantly with changes in worry symptoms (PSWQ, $r=-0.55, P=0.027$ ) and tended to correlate

\begin{tabular}{|c|c|c|c|c|}
\hline \multirow[b]{2}{*}{ Characteristic } & \multirow{2}{*}{$\begin{array}{l}\text { Active group } \\
\quad(n=13)\end{array}$} & \multirow{2}{*}{$\begin{array}{l}\text { Sham group } \\
\qquad(n=12)\end{array}$} & \multicolumn{2}{|c|}{ Statistical analysis $(n=25)$} \\
\hline & & & $t$-test (d.f.) & $\chi^{2}$ \\
\hline Age, mean (s.d.) & $44.00(11.95)$ & $44.58(14.75)$ & $0.11(23)$ & \\
\hline Women, $n$ (\%) & $11(84.6)$ & $8(66.7)$ & & $1.10(1)$ \\
\hline \multicolumn{5}{|l|}{ Ethnic status } \\
\hline White, $n(\%)$ & $12(92.3)$ & $12(100)$ & & $0.96(1)$ \\
\hline Non-Hispanic, $n$ (\%) & $12(92.3)$ & $12(100)$ & & $0.96(1)$ \\
\hline Education, high school diploma: $n$ (\%) & $12(92.3)$ & $12(100)$ & & $0.96(1)$ \\
\hline Employment, working: $n$ (\%) & $10(76.9)$ & $7(58.3)$ & & $0.99(1)$ \\
\hline Marital status, married: $n$ (\%) & $6(46.2)$ & $9(75.0)$ & & $2.16(1)$ \\
\hline Clinical Global Impression - severity scale, mean (s.d.) & $5.15(0.69)$ & $4.50(0.67)$ & $2.40 *(23)$ & \\
\hline Hamilton Rating Scale for Anxiety, mean (s.d.) & $25.31(5.23)$ & $20.75(3.72)$ & $2.49 *(23)$ & \\
\hline Penn State Worry Questionnaire, mean (s.d.) & $69.54(5.77)$ & $62.08(9.58)$ & $2.38 *(23)$ & \\
\hline Hamilton Rating Scale for Depression & $15.00(2.97)$ & $13.00(2.34)$ & $0.19(23)$ & \\
\hline Depression Anxiety Stress Scales, depression subscale: mean (s.d.) & $12.25(8.30)$ & $19.38(11.30)$ & $1.79(23)$ & \\
\hline Medication, taking psychotropic medications: $n$ (\%) & $9(69.2)$ & $8(66.7)$ & & $0.02(1)$ \\
\hline Any comorbid disorder, $n(\%)$ & $9(69.2)$ & $6(50.0)$ & & $0.96(1)$ \\
\hline Comorbid anxiety disorder, $n(\%)$ & $5(38.5)$ & $4(33.0)$ & & $0.07(1)$ \\
\hline Comorbid depressive disorder, $n$ (\%) & $8(61.5)$ & $3(25.0)$ & & 3.38 (1) \\
\hline
\end{tabular}




\begin{tabular}{|c|c|c|c|c|c|c|c|c|c|}
\hline \multirow[b]{2}{*}{$\begin{array}{l}\text { Treatment } \\
\text { group }\end{array}$} & \multicolumn{3}{|c|}{ Mean (s.d.) } & \multirow[b]{2}{*}{$F$, time } & \multirow{2}{*}{$\begin{array}{l}F \text {, group } \times \\
\text { time } \\
\text { interaction }\end{array}$} & \multirow{2}{*}{$\begin{array}{l}\text { Pre-to-post } \\
\text { treatment, } \\
t \text {-test }\end{array}$} & \multirow{2}{*}{$\begin{array}{c}\text { Pre-to-post } \\
\text { treatment, } \\
\text { Cohen's } d(95 \% \mathrm{Cl})\end{array}$} & \multirow{2}{*}{$\begin{array}{l}\text { Pre- } \\
\text { treatment to } \\
\text { follow-up, } t\end{array}$} & \multirow{2}{*}{$\begin{array}{l}\text { Pre-treatment to } \\
\text { follow-up Cohen's d } \\
(95 \% \mathrm{Cl})\end{array}$} \\
\hline & $\begin{array}{l}\text { Pre- } \\
\text { treatment }\end{array}$ & $\begin{array}{l}\text { Post- } \\
\text { treatment }\end{array}$ & Follow-up & & & & & & \\
\hline HRSA & & & & $36.56^{* * *}$ & $11.49 * * *$ & & & & \\
\hline Sham & $20.75(3.72)$ & $14.38(4.78)$ & $17.95(7.48)$ & & & $5.10^{* \star *}$ & 1.47 (0.63 to 2.29$)$ & 1.27 & $0.37(-0.23$ to 0.95$)$ \\
\hline Active & $25.31(5.23)$ & $12.10(5.77)$ & $10.36(7.86)$ & & & $6.90 * * *$ & $1.91(0.97-2.83)$ & $5.79 * * *$ & 1.61 (0.76 to 2.43$)$ \\
\hline PSWQ & & & & $16.77^{* * *}$ & $5.04^{*}$ & & & & \\
\hline Sham & $62.08(9.58)$ & $61.77(8.35)$ & $57.49(8.85)$ & & & 0.23 & $0.07(-0.50$ to 0.63$)$ & 2.15 & $0.62(-0.01$ to 1.23$)$ \\
\hline Active & $69.54(5.77)$ & $61.73(8.80)$ & $54.36(8.10)$ & & & $2.59 *$ & 0.72 (0.09 to 1.32 ) & $4.85^{\star * *}$ & 1.35 (0.57 to 2.09$)$ \\
\hline HRSD & & & & $9.06 * * *$ & $4.79 *$ & & & & \\
\hline Sham & $13.00(2.34)$ & $11.40(3.52)$ & $13.40(5.68)$ & & & 2.16 & $0.62(-0.01$ to 1.23$)$ & -0.28 & $-0.08(-1.04$ to 0.87$)$ \\
\hline Active & $15.00(2.97)$ & $9.30(4.39)$ & 7.78 (5.38) & & & $4.20 * *$ & 1.16 (0.44 to 1.86$)$ & $4.05^{\star \star}$ & 1.12 (0.41 to 1.81$)$ \\
\hline DASS-DEP & & & & $9.48^{* * *}$ & $4.79 *$ & & & & \\
\hline Sham & $12.25(8.30)$ & $10.49(6.66)$ & $10.02(11.21)$ & & & 1.37 & $0.39(-0.20$ to 0.98$)$ & 0.85 & 0.25 ( -0.33 to 0.82$)$ \\
\hline Active & & & & & & $2.68^{*}$ & 0.74 (0.11 to 1.35 ) & $3.17 *$ & 0.88 (0.22 to 1.51$)$ \\
\hline
\end{tabular}

with anxiety symptoms (HRSA, $r=-0.47, P=0.067$ ) such that greater symptom improvement was associated with greater increases in right DLPFC activation from pre- to post-treatment. Changes in right DLPFC activations were not associated with changes in depressive symptoms (HRSD, $r=-0.42, P=0.103$; DASS-DEP $r=-0.23, P=0.391)$.

\section{Discussion}

\section{Main finding and interpretation}

Results from this first RCT of neuromodulation in GAD provide preliminary evidence for the efficacy of rTMS. Pre-to-posttreatment effect sizes for symptom changes were uniformly larger in the active $v$. sham group. This interaction reached statistical significance for anxiety, worry and depressive symptoms. Response and remission rates were also higher in the active $v$. sham group. Only one prior open trial investigated rTMS in $\mathrm{GAD} ;{ }^{17}$ the outcomes of that study (i.e. high response rates and large pre-to-post anxiolytic effects) were similar to those of the current study. The same stimulation parameters were used (lowfrequency right DLPFC), however, the current protocol entailed a higher number and frequency of rTMS sessions culminating in a net gain of 21600 total pulses. As of yet the optimal treatment parameters for GAD are not known. In the current study, the intense treatment schedule was a common reason for refusal and withdrawal, and many enrolled participants experienced difficulty complying with the schedule. It will be important to identify efficacious dosing schedules that are more acceptable and feasible, such as accelerated rTMS administered over a course of a few days (see for example McGirr et $a l^{31}$ ).

The optimal stimulation target for treating GAD is also unknown. Support for the DLPFC comes from anxiolytic effects of rTMS in patients with major depressive disorder ${ }^{10}$ and changes in anxiety-related biological processes in healthy controls (see for example Baeken et $a l^{16}$ ). The emotion dysregulation model of GAD provides a theoretical rationale for DLPFC stimulation. Emotion regulation is the process of identifying and altering emotional experiences, and GAD patients demonstrate problems with these skills. ${ }^{32}$ The DLPFC plays a central role in emotion regulation processes via its connections with cortical and subcortical regions (for example dorsal anterior cingulate cortex, inferior frontal gyrus, ventral anterior cingulate cortex, VMPFC). In particular the connection with the VMPFC may mediate DLPFC stimulation and limbic activations. ${ }^{33}$ Neuromodulation of the DLPFC may therefore improve emotion regulation of anxiety by having an impact on the functioning of and/or communication within these frontolimbic networks.

In the current study a treatment course of low-frequency (inhibitory) stimulation of the right DLPFC was associated with increased activation in the target site during decision-making and neural activation changes were associated with changes in worry. While engaging in emotion regulation tasks, patients with GAD demonstrate hypoactivation in the PFC as well as the anterior cingulate cortex and decreased structural and functional connectivity between frontal and limbic regions. ${ }^{11}$ These abnormalities may reflect deficient neurobiological 'top-down' emotional control. Given that these abnormalities are

Table 3 Frequency of patients reporting adverse events at any time point

\begin{tabular}{|c|c|c|c|}
\hline & \multicolumn{2}{|c|}{$n(\%)$} & \multirow[b]{2}{*}{$\chi^{2}$ (d.f.) $(n=25)$} \\
\hline & Active group $(n=13)$ & Sham group $(n=12)$ & \\
\hline Pin prick sensation & $9(69.2)$ & $10(83.3)$ & $0.68(1)$ \\
\hline Pain at the stimulation site & $11(84.6)$ & $8(66.7)$ & $1.10(1)$ \\
\hline Facial pain (including eye pain) & $3(23.1)$ & $1(8.3)$ & $1.01(1)$ \\
\hline Headache & $6(46.2)$ & $3(25.0)$ & $1.21(1)$ \\
\hline Toothache & $3(23.1)$ & $0(0)$ & $3.15(1)$ \\
\hline Lightheaded or dizziness & $0(0)$ & $2(16.7)$ & $2.36(1)$ \\
\hline Facial twitch & $6(46.2)$ & $0(0)$ & $7.29 *(1)$ \\
\hline
\end{tabular}


characterised in part by DLPFC hypoactivation in patients with $\mathrm{GAD},{ }^{34}$ results from the current study are suggestive of DLPFC normalisation over treatment, which enhances top-down regulation over prefrontal and limbic areas. Improvements in GAD symptoms following pharmacotherapy or counselling also demonstrate normalisation in DLPFC activation, ${ }^{35}$ as well as improved connectivity between the DLPFC and other prefrontal regions ${ }^{36}$ and between the $\mathrm{PFC}$ and amygdala. ${ }^{37}$ Connectivity analyses of data from the current study are in process and may further elucidate the effect of rTMS treatment on GAD neurocircuitry.

\section{Strengths and limitations}

The current RCT is a substantial advancement and critical step toward empirically supporting rTMS for GAD. However, results should be considered preliminary because of the small sample sizes. Attrition rates were also higher than those in rTMS trials for major depressive disorder ${ }^{8}$ but in-line with pharmacotherapy trials in $\mathrm{GAD}^{38}$ Adverse events were largely similar between treatment conditions; however, facial twitch was more common in the active group. It will be important for future research to minimise this potential threat to unmasking (for example, by using a protocol to prevent disclosure of facial twitch to evaluators). In addition, the randomisation schedule did not equally distribute anxiety symptoms, with those patients with more severe anxiety being allocated to active treatment. However, the treatment effect in active rTMS was not consistent with a regression to the mean interpretation, as patients who benefitted typically reported symptoms within or close to remission. Although active treatment was superior to sham, there was a large acute anxiolytic effect in sham as well. Individuals with GAD are prone to placebo response, ${ }^{39}$ and the effect size in the current study is consistent with the large placebo effect for neuromodulation found in patients with major depressive disorder. ${ }^{40}$ Importantly, improvements in the sham group were not maintained whereas patients receiving active rTMS tended to maintain or improve over follow-up. In a previous open trial rTMS outcomes were maintained over 6 months. ${ }^{18}$ However, long-term durability is not known and future research will need to investigate relapse risk and the potential use of maintenance rTMS as is often done clinically for patients with major depressive disorder.

It will also be important for future research to establish rTMS mechanisms of action. Data from the current study are informative as the first to report on neurobiological changes following rTMS treatment for GAD. However, the biological process underlying the mechanism by which inhibitory stimulation led to increased DLPFC activation during decisionmaking is not clear. It is hypothesised that excessive inhibition within the frontolimbic network is subsequently normalised after treatment, but this is purely speculative. It will also be important to explore the biological mechanisms of anxiety improvements following rTMS such as normalisation of neuroendocrine, neurotransmitter and/or neurotrophic factors. ${ }^{15}$ This research will be facilitated by investigating the impact and predictors of neuromodulation on transdiagnostic biological and behavioural constructs consistent with the National Institute of Mental Health Research Domain Criteria initiative. ${ }^{41}$ Such efforts will provide the foundation for more personalised and targeted neuromodulation treatments in the future.

\section{Funding}

This study was funded by a grant (number: 129522) from the Hartford HealthCare Research Funding Initiative to G.J.D. The funding source had no role in the study design; collection, analysis, interpretation of data; writing the report, or in making the decision to submit the article for publication. Material support was provided by Neuronetics. Neuronetics reviewed a draft of this report prior to submission and otherwise had no role in the study design; collection, analysis, interpretation of data; writing the report, or in making the decision to submit the article for publication.

\section{Acknowledgements}

We thank Joost van Ginkel for providing an SPSS macro and providing consultation on multiple imputation procedures.

Gretchen J. Diefenbach, PhD, Anxiety Disorders Center, The Institute of Living, Hartford and Yale University School of Medicine, New Haven, Connecticut; Laura B. Bragdon, MA, Binghamton University, Binghamton, New York; Luis Zertuche, BSC, Bragdon, MA, Binghamton University, Binghamton, New York; Luis Zertuche, BSc,
Christopher J. Hyatt, PhD, Olin Neuropsychiatry Research Center, The Institute of Living, Hartford, Connecticut; Lauren S. Hallion, PhD, Anxiety Disorders Center, The Institute of Living, Hartford, Connecticut; David F. Tolin, PhD, Anxiety Disorders Center, The Institute of Living, Hartford and Yale University School of Medicine, New Haven, Connecticut; John W. Goethe, MD, Burlingame Center for Research and Education, The Institute of Living, Hartford, Connecticut; Michal Assaf, MD, Olin Neuropsychiatry Research Center, The Institute of Living, Hartford and Yale University School of Medicine, New Haven, Connecticut, USA

Correspondence: Gretchen J. Diefenbach, PhD, Anxiety Disorders Center The Institute of Living, 200 Retreat Avenue, Hartford, CT 06106, USA. Email: Gretchen.Diefenbach@hhchealth.org

First received 20 Apr 2015, final revision 19 Aug 2015, accepted 27 Sep 2015

\section{References}

1 American Psychiatric Association. Diagnostic and Statistical Manual of Mental Disorder (5th edn) (DSM-5). APA, 2013

2 Kessler RC, Berglund P, Demler O, Jin R, Walters EE. Lifetime prevalence and age-of-onset distributions of DSM-IV disorders in the National Comorbidity Survey Replication. Arch Gen Psychiatry 2005; 62: 593-602.

3 Revicki DA, Travers K, wyrwich KW, Svedsater H, Locklear J, Mattera MS, et al. Humanistic and economic burden of generalized anxiety disorder in North America and Europe. J Affect Disord 2012; 140: 103-12.

4 Lydiard RB, Monnier J. Pharmacological treatment. In Generalized Anxiety Disorder: Advances in Research and Practice (eds RG Heimberg, CL Turk, DS Mennin): 351-79. Guilford Press, 2004

5 Huppert JD, Sanderson WC. Psychotherapy for generalized anxiety disorder In Textbook of Anxiety Disorders (eds DJ Stein, E Hollander, BO Rothbaum): 219-38. American Psychiatric Publishing, 2010

6 Tracy DK, de Sousa de Abreu M, Nalesnik N, Mao L, Lage C, Shergill SS Neuroimaging effects of $1 \mathrm{~Hz}$ right temporoparietal rTMS on normal auditory processing: implications for clinical hallucination treatment paradigms. J Clin Neurophysiol 2014; 31: 541-6.

7 Siebner HR, Hartwigsen G, Kassuba T, Rothwell JC. How does transcranial magnetic stimulation modify neuronal activity in the brain? Implications for studies of cognition. Cortex 2009; 45: 1035-42.

8 Berlim MT, van den Eynde F, Tovar-Perdomo S, Daskalakis ZJ. Response, remission and drop-out rates following high-frequency repetitive transcranial magnetic stimulation (rTMS) for treating major depression: a systematic review and meta-analysis of randomized, double-blind and sham-controlled trials. Psychol Med 2014; 44: 225-39.

9 Berlim MT, Van den Eynde F, Jeff Daskalakis Z. Clinically meaningful efficacy and acceptability of low-frequency repetitive transcranial magnetic stimulation (rTMS) for treating primary major depression: a meta-analysis of randomized, double-blind and sham-controlled trials. Neuropsychopharmacology 2013; 38: 543-51.

10 Diefenbach GJ, Bragdon LB, Goethe JW. Treating anxious depression using repetitive transcranial magnetic stimulation. J Affect Disord 2013; 151 365-8.

11 Mochcovitch MD, da Rocha Freire RC, Garcia RF, Nardi AE. A systematic review of fMRI studies in generalized anxiety disorder: evaluating its neural and cognitive basis. J Affect Disord 2014; 167: 336-42.

12 Hilbert $\mathrm{K}$, Lueken $\mathrm{U}$, Beesdo-Baum $\mathrm{K}$. Neural structures, functioning and connectivity in Generalized Anxiety Disorder and interaction with neuroendocrine systems: a systematic review. J Affect Disord 2014; 158 $114-26$.

13 Paulesu E, Sambugaro E, Torti T, Danelli L, Ferri F, Scialfa G, et al. Neura correlates of worry in generalized anxiety disorder and in nomral controls: a functional MRI study. Psychol Med 2010; 40: 117-24. 
14 Baumgartner T, Knoch D, Hotz P, Eisenegger C, Fehr E. Dorsolateral and ventromedial prefrontal cortex orchestrate normative choice. Nature Neurosci 2011; 14: 1468-74.

15 Baeken C, De Raedt R. Neurobiological mechanisms of repetitive transcrania magnetic stimulation on the underlying neurocircuitry in unipolar depression. Dialogues Clin Neurosci 2011; 13: 139-45.

16 Baeken C, Vanderhasselt MA, De Raedt R. Baseline 'state anxiety' influences HPA-axis sensitivity to one sham-controlled HF-rTMS session applied to the right dorsolateral prefrontal cortex. Psychoneuroendocrinology 2011; 36: 60-7.

17 Bystritsky A, Kaplan JT, Feusner JD, Kerwin LE, Wadekar M, Burock M, et al. A preliminary study of $\mathrm{fMRI}$-guided rTMS in the treatment of generalized anxiety disorder. J Clin Psychiatry 2008; 69: 1092-8.

18 Bystritsky A, Kerwin LE, Feusner JD. A preliminary study of fMRI-guided rTMS in the treatment of generalized anxiety disorder: 6-month follow-up. J Clin Psychiatry 2009; 70: 431-2.

19 Dugas MJ, Gagnon F, Ladouceur R, Freeston MH. Generalized anxiety disorder: a preliminary test of a conceptual model. Behav Res Ther 1998; 36 215-26.

20 Guy W. Assessment Manual for Psychopharmacology. US Government Printing Office, 1976.

21 Shear MK, Vander Bilt J, Rucci P, Endicott J, Lydiard R, Otto MW, et al. Reliability and validity of a structured interview guide for the Hamilton Anxiety Rating Scale (SIGH-A). Depress Anxiety 2001; 13: 166-78.

22 Hamilton M. A rating scale for depression. J Neurol Neurosurg Psychiatry 1960; 23: 56-62.

23 Sheehan DV, Lecrubier $\mathrm{Y}$, Sheehan $\mathrm{KH}$, Amorim $\mathrm{P}$, Janavs J, Weiller $\mathrm{E}$, et al. The Mini-International Neuropsychiatric Interview (MINI): the development and validation of a structured diagnostic psychiatric interview for DSM-IV and ICD-10. J Clin Psychiatry 1998; 59 (suppl 20): 22-33.

24 Brown TA, Di Nardo PA, Lehman CL, Campbell LA. Reliability of DSM-IV anxiety and mood disorders: Implications for the classification of emotional disorders. J Abnorm Psychol 2001; 110: 49-58.

25 Meyer TJ, Miller ML, Metzger RL, Borkovec TD. Development and validity of the Penn State Worry Questionnaire. Behav Res Ther 1990; 28: 487-95.

26 Lovibond SH, Lovibond PF. Manual for the Depression Anxiety Stress Scales. Psychology Foundation of Australia, 1995.

27 Williams JB. A structured interview guide for the Hamilton Depression Rating Scale. Arch Gen Psychiatry 1988; 45: 742-7.

28 Rubin DB. Multiple Imputation for Nonresponse in Surveys. J Wiley \& Sons, 1987.
29 Cohen J. Statistical Power Analysis for the Behavioral Sciences, 2nd edn Lawrence Erlbaum Associates, 1988.

30 Nielsen FA, Hansen LK. Modeling of activation data in the BrainMap database: detection of outliers. Hum Brain Mapp 2002; 15: 146-56.

31 McGirr A, Van den Eynde F, Tovar-Perdomo S, Fleck MP, Berlim MT. Effectiveness and acceptability of accelerated repetitive transcranial magnetic stimulation (rTMS) for treatment-resistant major depressive disorder: an open label trial. J Affect Disord 2015; 173: 216-20.

32 Mennin DS, Heimberg RG, Turk CL, Fresco DM. Preliminary evidence for an emotion dysregulation model of generalized anxiety disorder. Behav Res Ther 2005; 43: 1281-310.

33 Diekhof EK, Geier K, Falkai P, Gruber O. Fear is only as deep as the mind allows: a coordinate-based meta-analysis of neuroimaging studies on the regulation of negative affect. Neuroimage 2011; 58: 275-85.

34 Ball TM, Ramsawh HJ, Campbell-Sills L, Paulus MP, Stein MB. Prefrontal dysfunction during emotion regulation in generalized anxiety and panic disorders. Psychol Med 2013; 43: 1475-86.

35 Hoehn-Saric R, Schlund MW, Wong SH. Effects of citalopram on worry and brain activation in patients with generalized anxiety disorder. Psychiat Res 2004; 131: 11-21.

36 Andreescu C, Sheu LK, Tudorascu D, Gross JJ, Walker S, Banihashemi L, et al. Emotion reactivity and regulation in late-life generalized anxiety disorder: functional connectivity at baseline and post-treatment. Am J Geriatr Psychiatry 2015; 23: 200-14

37 Maslowsky J, Mogg K, Bradley BP, McClure-Tone E, Ernst M, Pine DS, et al. A preliminary investigation of neural correlates of treatment in adolescents with generalized anxiety disorder. J Child Adolesc Psychopharmacol 2010; 20: 105-11.

38 Mitte K. Meta-analysis of cognitive-behavioral treatments for generalized anxiety disorder: a comparison with pharmacotherapy. Psychol Bull 2005; 131: 785-95.

39 Khan A, Kolts RL, Rapaport MH, Krishnan KR, Brodhead AE, Browns WA. Magnitude of placebo response and drug-placebo differences across psychiatric disorders. Psychol Med 2005; 35: 743-9.

40 Brunoni AR, Lopes M, Kaptchuk TJ, Fregni F. Placebo response of nonpharmacological and pharmacological trials in major depression: a systematic review and meta-analysis. PLoS One 2009; 4: e4824.

41 Cuthbert BN. The RDoC framework: facilitating transition from ICD/DSM to dimensional approaches that integrate neuroscience and psychopathology. World Psychiatry 2014; 13: 28-35. 